\title{
A Simple Magnetostatic Sensing Method for Assessing the Local Hysteresis Properties in Ferromagnetic Sheet Materials
}

\author{
Ivan J. Garshelis ${ }^{1}$ and Guillaume Crevecoeur ${ }^{2}$ \\ ${ }^{1}$ Magnova, Inc., 17 Downing Three, Pittsfield, MA 01201, USA \\ ${ }^{2}$ Department Electrical Energy, Systems and Automation, Ghent University, Sint-Pietersnieuwstraat 41, B-9000 Ghent, Belgium
}

Correspondence should be addressed to Ivan J. Garshelis, ijgarsh@att.net

and Guillaume Crevecoeur, guillaume.crevecoeur@ugent.be

Received 4 November 2011; Accepted 8 March 2012

Academic Editor: Lalita Udpa

Copyright (C) 2012 I. J. Garshelis and G. Crevecoeur. This is an open access article distributed under the Creative Commons Attribution License, which permits unrestricted use, distribution, and reproduction in any medium, provided the original work is properly cited.

\begin{abstract}
Global hysteretic properties of electrical steels can be measured using ring or strip samples, while the assessment of the local hysteretic properties is a much more difficult task since the measurement method needs to be very sensitive. This paper presents a new method wherein the intensity and spatial distribution of the magnetic field, arising from large gradients in the local magnetization, are measured. These large gradients are induced by the passage of a test sample through the steep gradient field of a small, proximate permanent magnet. Magnetic field measurements during both directions of motion provide information indicative of the hysteresis properties. We theoretically analyze these measurements and show experimentally that the measurements correlate well with all the significant aspects of conventional hysteresis loops. The results given in this paper are qualitative, and the method is both by its simplicity and its sensitivity to important hysteresis features a powerful means of magnetic nondestructive evaluation.
\end{abstract}

\section{Introduction}

Hysteresis loss is not only a critical factor in the selection of steels for use in electrical machines but, by its nature, can also provide significant information on the structural condition and/or magnetic anisotropy of ferromagnetic materials generally. With electrical steels, the energy loss itself is the usual parameter of prime interest, whereas one or both of its (typically) key components, namely, coercivity and the remanent induction, or at some other "standard" field intensity, provide the sought for information. There is a need for a technique that can give useful information about relationships between microstructure or internal stress with magnetic performance.

Conventional measurements of hysteretic properties usually employ ring or strip samples, with the latter being necessary when anisotropy is being explored [1]. Indeed, the directional nonuniformity of the magnetic properties is measured by cutting samples at various angles from a sheet of material. Nondestructive determinations of local properties on the other hand, for example, to explore the relative structural damage associated with different processes used to cut electrical steel sheets, typically require either specially prepared samples [2], sophisticated techniques such as the needle probe method [3], or specialized apparatus such as with the drag force method [4]. All methods except drag force employ magnetic fields derived from electric currents.

A new magnetostatic method for obtaining comparative measurements of hysteresis loss and its components in ferromagnetic sheet materials is described in this paper. The measurements will be shown to provide qualitative information, which correlates directly with all of the significant aspects of conventional hysteresis loops. Essential features of the measurement apparatus are shown in Figure 1. The distance between the Motion Limits is called the "Stroke." The field sensor is located at the center of the Stroke, generally as close to the SUT surface as its physical package allows $(c \approx 0)$. The size of the Stroke is typically from $10 \mathrm{G}$ to $20 \mathrm{G}$. Remark that the presented method can measure the directional nonuniformity of the magnetic properties in a nondestructive way. 


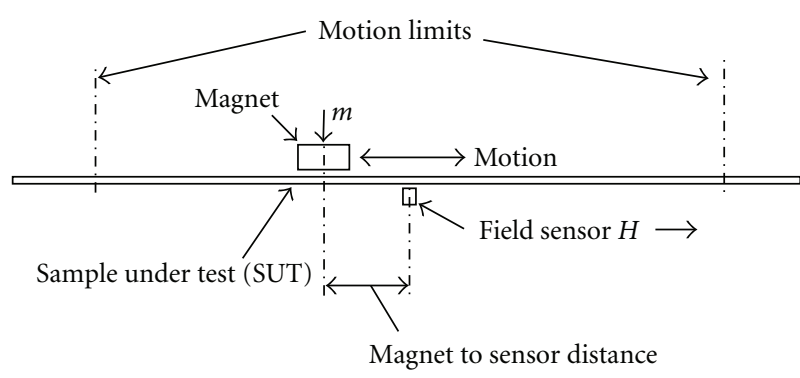

(a)

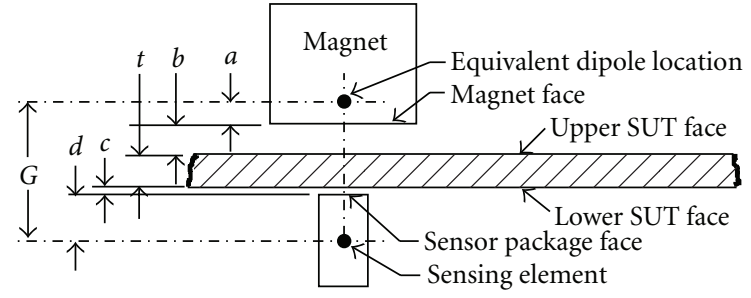

(b)

Figure 1: (a) Side view of basic arrangement. (b) Magnified view of the active elements showing the dimensional factors that contribute to the "Gap", $G \cdot x$ is the longitudinal distance between the magnet and the field sensor.

The modus operandi of the measurement method is as follows: the magnet, polarized normal to the sample under test (SUT) surface, initially located at one end of the Stroke is moved, at a speed slow enough $(\sim 3 \mathrm{~mm} / \mathrm{s})$ such that making it slower does not significantly alter the results, across the face of the SUT at a small fixed separation distance $(0.05-$ $2.5 \mathrm{~mm}$ ) until it reaches the opposite Motion Limit at which time the motion is stopped. It is then moved, at the same speed and separation distance, back to its starting position. This "initializing" cycle of motion is repeated several times to obtain stable magnetization excursions in the SUT. During the next forward traversal of the Stroke, both the intensity of the field (in the longitudinal direction), $H_{\mathrm{F}}(x)$, and the coincidental longitudinal distance between the magnet and the field sensor, $x$, are measured and recorded. After a momentary stop at the end of the Stroke, motion is resumed in the reverse direction, during which, the field, $H_{\mathrm{R}}(x)$, and corresponding position are again measured and recorded. For each recorded data pair $(H(x), x)$, the difference, $H_{\mathrm{F}}(x)-$ $H_{\mathrm{R}}(x)=D(x)$, is calculated. As will be seen, $D(x)$ will have some salient features (maxima, minima, and others) that correlate with the SUT's hysteresis loss and its components.

\section{Theory}

2.1. Assumptions. The inarguable simplicity of the method and apparatus notwithstanding, a detailed understanding of its operation is quite the opposite. Nevertheless, the operational basis and a qualitative relationship between features of $D(x)$ and hysteresis can be made adequately clear by an analytical model built from the following assumptions.

(1) The existence of the signature features in $D(x)$ reflects magnetic hysteresis in the sample; in the absence of hysteresis $D(x) \equiv 0$.

(2) The field from the magnet and its spatial distribution are approximated well enough by that of an equal moment dipole located within the magnet body.

(3) A solution of the $2 \mathrm{D}$ problem is sufficient.

(4) The sample is assumed to be thin enough such that the field from the magnet, while varying with longitudinal position, is uniform throughout its thickness. This assumption ignores thus also radial components of the field from the magnet.

(5) The instantaneous local magnetization at points within the sample, $M(x)$, is determined entirely by the instantaneous local field from the magnet, $H_{L}(x)$, and the history of changes in that field. It is recognized that $H_{\mathrm{F}}(x)$ and $H_{\mathrm{R}}(x)$ are each comprised of components from two sources, the magnet and $\nabla \cdot M(x)$ within the sample. Nevertheless, effects of these latter fields on $M(x)$ are ignored on the assumption that they modify only details of the $D(x)$ signature features, not their presence.

(6) The $M-H$ characteristics of the sample can be defined by any function which results in closed, symmetrical, sigmoidal loops without concern for underlying physical sources, for example, domain wall pinning or anisotropy. Thus suitable loops can be constructed from purposefully modified Langevin functions.

The authors are fully aware that this model is an approximation, but it enables to directly correlate simple hysteresis properties with the $D(x)$ measurements. This is the major aim of the theory shown here. More advanced numerical techniques could be used for understanding in depth the measurements.

2.2. Analysis. Guided by the previous assumptions, the analysis proceeds as follows.

(a) Determine the variation with $x$ of the longitudinal component of the field $\left(H_{L}\right)$ from the equivalent dipole source.

(b) Determine the sequence of field variation at underlying points in the sample during forward and reverse motion of the magnet, that is, $H_{L}(x)_{\mathrm{F}}$ and $H_{L}(x)_{\mathrm{R}}$.

(c) Create families of hysteresis loops with variable loss densities and components, that is, $M\left(H_{L}\right)$ functions with different values of coercivity and remanence.

(d) Determine $M(x)$ for both directions of motion by transposing the sequence of field variation, that is, $H_{L}(x)_{\mathrm{F}}$ and $H_{L}(x)_{\mathrm{R}}$ onto the $M\left(H_{L}\right)$ loops created in (c). 
(e) Determine $\nabla \cdot M(x)$ (= $\varphi$, the free-pole density) for each direction of motion. (This becomes a second source (in addition to $m$ ) of the sensed field.)

(f) Determine $\nabla \varphi(x)=-H$ for each direction of motion, that is, $H_{\mathrm{F}}(x)$ and $H_{\mathrm{R}}(x)$.

(g) Determine $D(x)=H_{\mathrm{F}}(x)-H_{\mathrm{R}}(x)$, identify, and characterize salient features.

(h) Correlate feature characterizations from (g) with hysteresis loop features from (c).

For the arrangement diagrammed in Figure 2, the relationship between $H_{L}$ at point $P$ (the location of the field sensor in Figure 1), and $P$ 's distance from a dipole of moment $m$, is found (following from Cullity's derivation [5]) as

$$
H_{L}=3 m \frac{x G}{\left(x^{2}+G^{2}\right)^{5 / 2}} \text {. }
$$

The longitudinal component $H_{L}$ is directed to the right at points to the right of $O(x>0)$ and to the left at points to the left of $O(x<0)$, thus $H_{L}(x)=-H_{L}(-x)$. Equation (1) with $m=1$ and $G=1$ is plotted in Figure 3. The numbers indicate noteworthy magnet (dipole) positions relative to a field sensor at $x=0$ during its motion in the forward, $\mathrm{F}$, and reverse, $\mathrm{R}$, directions of motion. When, for example, the magnet is at position $1, H_{L 1}=-0.00435$, at position 2, $H_{L 2}=-0.8587$, at position $3, H_{L 3}=0$, and so forth. Since the field distribution is effectively "attached" to the magnet, it moves together with the magnet. Determination of $M(x)$ requires knowledge of the corresponding $M-H$ relationships for the sample material. Assumption 6 permits suitable, analytically defined $M-H$ functions to be created from modified Langevin functions.

Ascending and descending limbs of hypothetical hysteresis loops are, respectively, generated from

$$
\begin{aligned}
& M_{a}=\operatorname{coth}\left(k\left(H-H_{c}\right)\right)-\frac{1}{k\left(H-H_{c}\right)}, \\
& M_{d}=\operatorname{coth}\left(k\left(H-H_{c}\right)\right)-\frac{1}{k\left(H+H_{c}\right)},
\end{aligned}
$$

wherein the "constants" $H_{c}$ and $k$ provide means to vary the "coercive field" and "remanence ratio," respectively, thereby to simulate different sample materials. Indeed, we use $H_{c}$ to shift the function along the $H$ axis, to the left $\left(+H_{c}\right)$ to obtain the descending limb of the loop and to the right $\left(-H_{c}\right)$ to obtain the ascending limb. Similarly, we use $k$ to adjust the tilt of these loop limbs, which modifies the remanence ratio.

A closed loop is formed by shifting $M_{a}$ upward and $M_{d}$ downward by $1 / 2$ the difference, $\Delta=M_{d}-M_{a}$, when $H$ is at its peak value, $H_{P}$, in (2) and (3). The loop will then consist of an ascending limb:

$$
M_{A}=M_{a}+\frac{\Delta}{2},
$$

and a descending limb:

$$
M_{D}=M_{d}-\frac{\Delta}{2}
$$

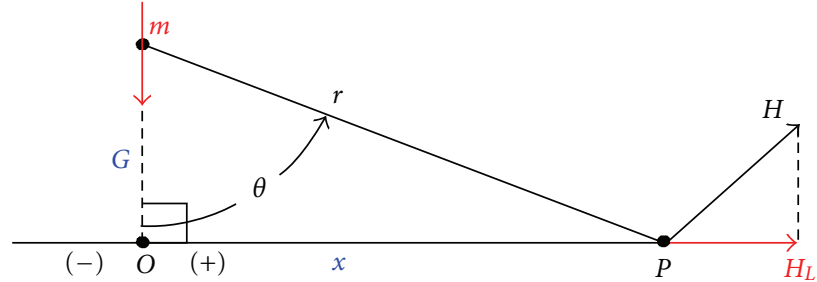

Figure 2: Diagram showing the field $H$, present at a point $P$, distance $r$, and angle $q$ ( $x$ and $G$ ) from dipole $m$.

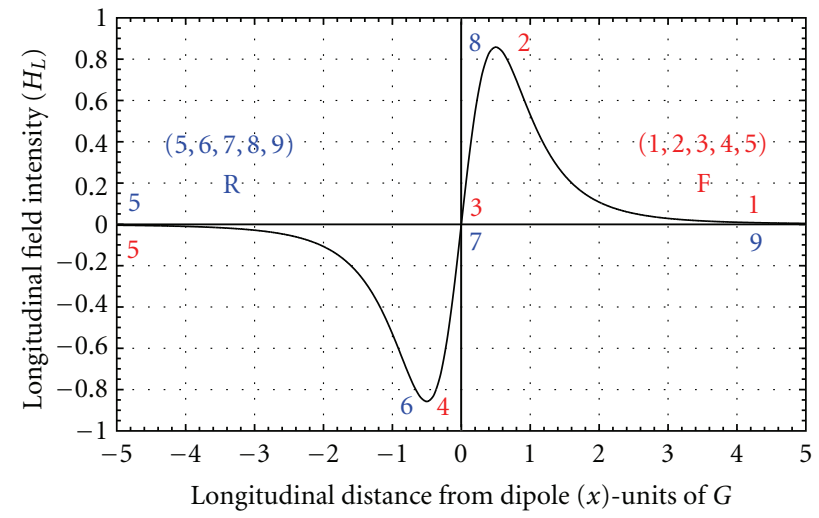

FIGURE 3: Variation in (normalized) $H_{L}$ with distance $x$. Note that $H_{L}$ is symmetrical around the origin, has peak values of \pm 0.8587 at $x= \pm 0.5$, and becomes only \pm 0.00435 at $x= \pm 5$.

Hypothetical positive and negative minor limbs from remanence $(H=0)$ to $H= \pm H_{P}$ can be produced from

$$
\begin{aligned}
& M_{m+}=M_{A}+\frac{M_{D}-M_{A}}{1+f^{*} H}, \\
& M_{m-}=M_{D}+\frac{M_{A}-M_{D}}{1-f^{*} H},
\end{aligned}
$$

where $f(\ll 1)$ is selected to insure that $\left|M_{m}\right|$ increases continuously with increasing $|H|$ while staying always between $M_{A}$ and $M_{D}$. Of the three shape modifiers $\left(H_{c}, k\right.$, and $f$ ), only $H_{c}$ has physical dimensions (the same as those of $H$ ).

From the above equations, it is possible to determine $\nabla \cdot M(x)=\varphi$ for both the forward and reverse directions: $\varphi_{\mathrm{F}}=\left(\partial M_{m+}(x)\right) / \partial x$ and $\varphi_{\mathrm{R}}=\left(\partial M_{m-}(x)\right) / \partial x$, from which $H_{\mathrm{F}}(x)=\nabla_{\varphi \mathrm{F}}(x)$ and $H_{\mathrm{R}}(x)$, and thus $D(x)$, can be calculated.

To avoid the need to deal with equations having ever growing numbers of ever more complex terms, and to provide means for graphically following the evolving analysis, we assign arbitrary (but as will be seen, arguably reasonable) values to the material-dependent parameters $H_{c}, k$, and $f$, and set $H_{P}=15$. In this way, $M_{A}, M_{D}, M_{m+}\left(\right.$ for $\left.0 \leq H \leq H_{P}\right)$, and $M_{-m}$ (for $0 \geq H \geq-H_{P}$ ) become numerical functions of $H$. For similar reasons and to make $H_{L \text { Peak }}=H_{P}=15$, we assign the value $5 / 0.8587=17.47$ to $m$ in (1).

As the magnet moves forward from $x=-5$ to $x=$ 5, $H_{L}(0)$ and $M(0)$ will follow the sequence shown in Figure 4(a), and during reverse motion (from $x=5$ to $x=$ $-5)$, the sequence shown in Figure 4(b). The corresponding 


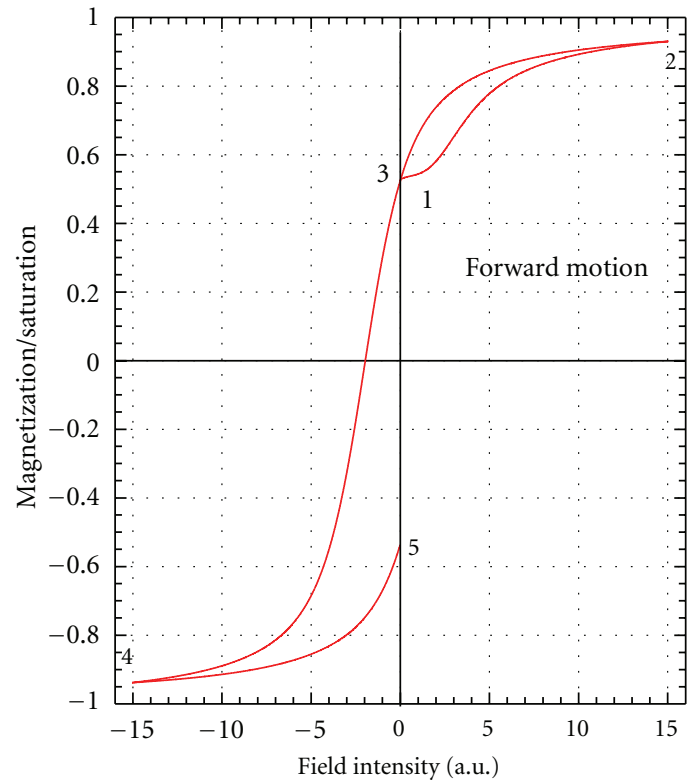

(a)

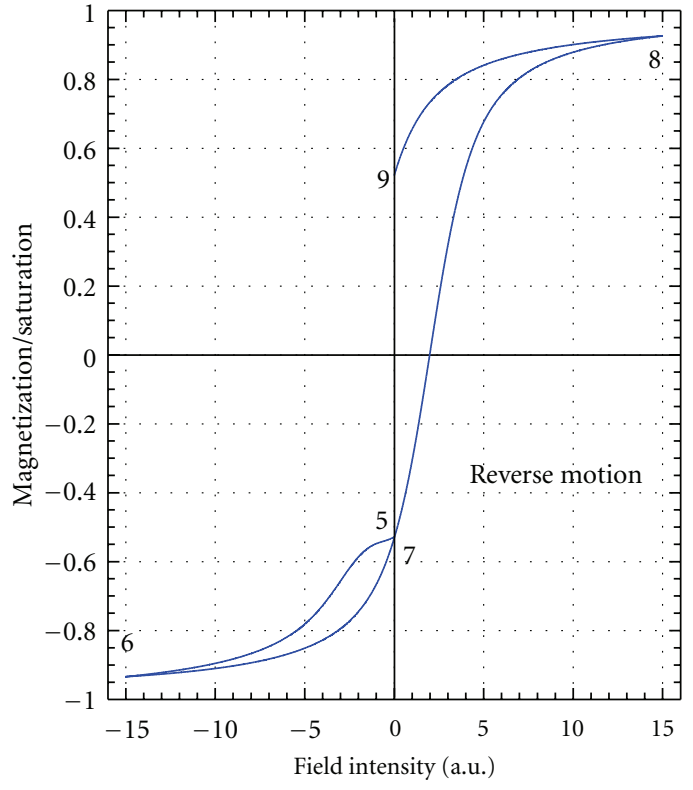

(b)

FIGURE 4: (a) Variation of field and resulting magnetization at the point on the SUT, which is directly over the field sensor during forward motion of the magnet. (b) Same for reverse motion. $\left(H_{c}=2, k=1, f=0.135\right)$.

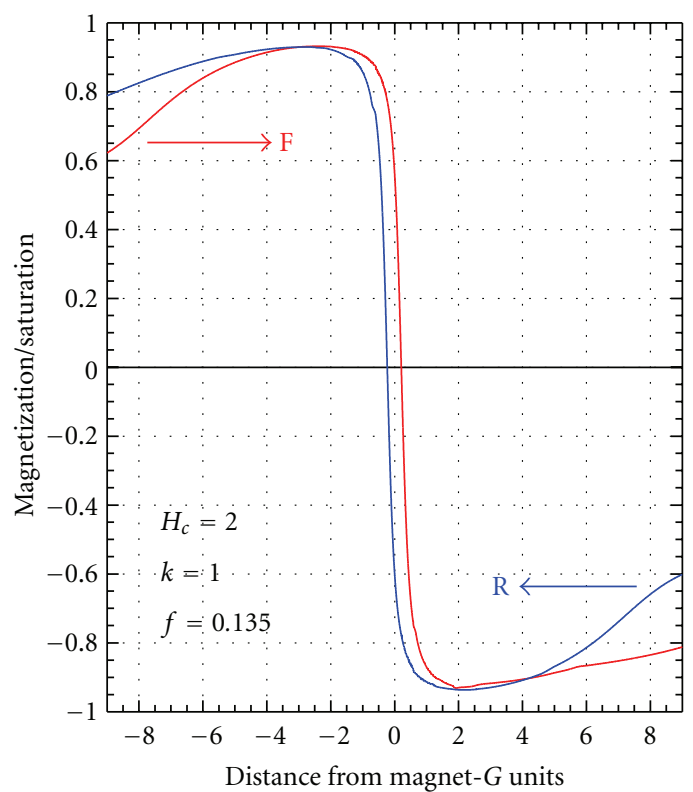

(a)

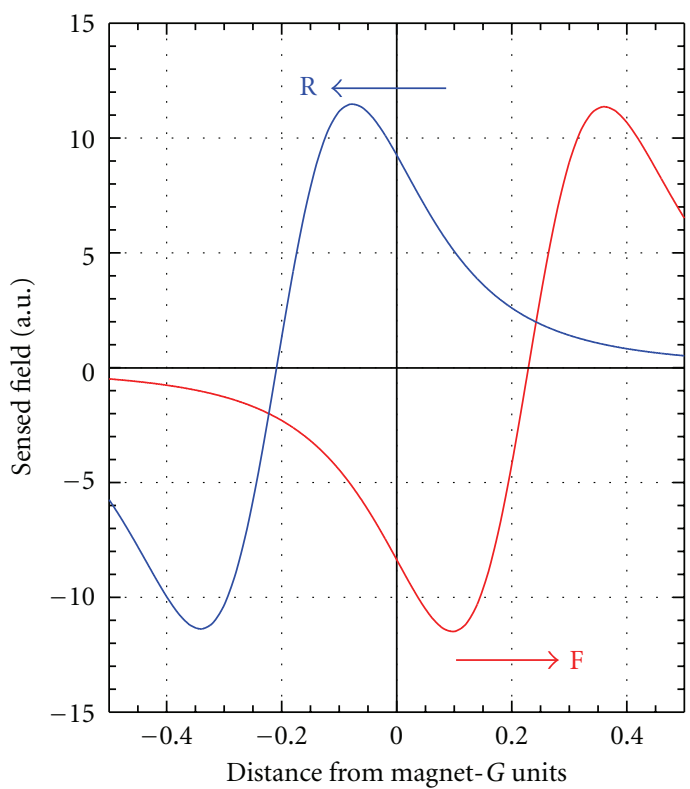

(b)

Figure 5: (a) Variation of $M$ at the sensor position during forward (F) and reverse (R) motion. (b) Corresponding sensed field $=\nabla(\nabla$. $\left.M(0)_{\mathrm{F}}\right)$ and $\nabla\left(\nabla \cdot M(0)_{\mathrm{R}}\right)$.

variations of $M(0)_{\mathrm{F}}$ and $M(0)_{\mathrm{R}}$ with magnet position are shown in Figure 5(a), with the central regions of $\nabla(\nabla$. $\left.M(0)_{\mathrm{F}}\right)$ and $\nabla\left(\nabla \cdot M(0)_{\mathrm{R}}\right)$ shown in Figure $5(\mathrm{~b})$. The starting points in Figure 5(a) are not exactly the same as the numbered points in Figure 4. This is because the field at the end of the motion in either direction does not reach zero at any finite values of $x$. Figure 4(a) presumes that the value of $x$ at the motion extrema is large enough for the field starting and finishing points to be close enough to zero. In contrast, the start (and finish) of the plots (but not the motion) in Figure 5 clearly occur at finite $( \pm 8.8 \mathrm{G})$ distances from the sensor. Thus the end of the plot in one direction does not have the same magnetization as the start of the plot in the opposite direction. 


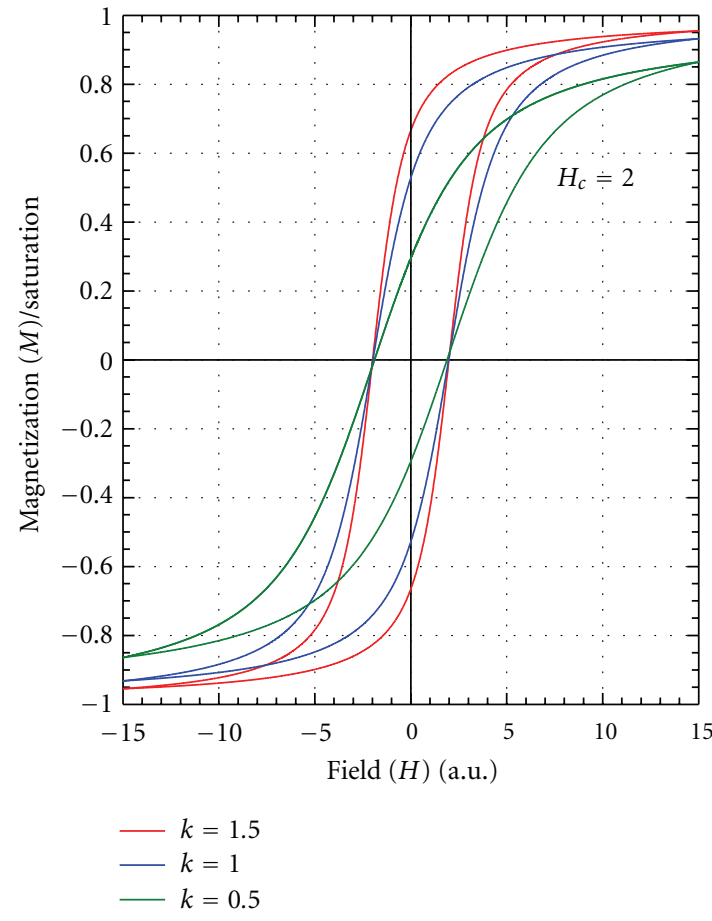

(a)

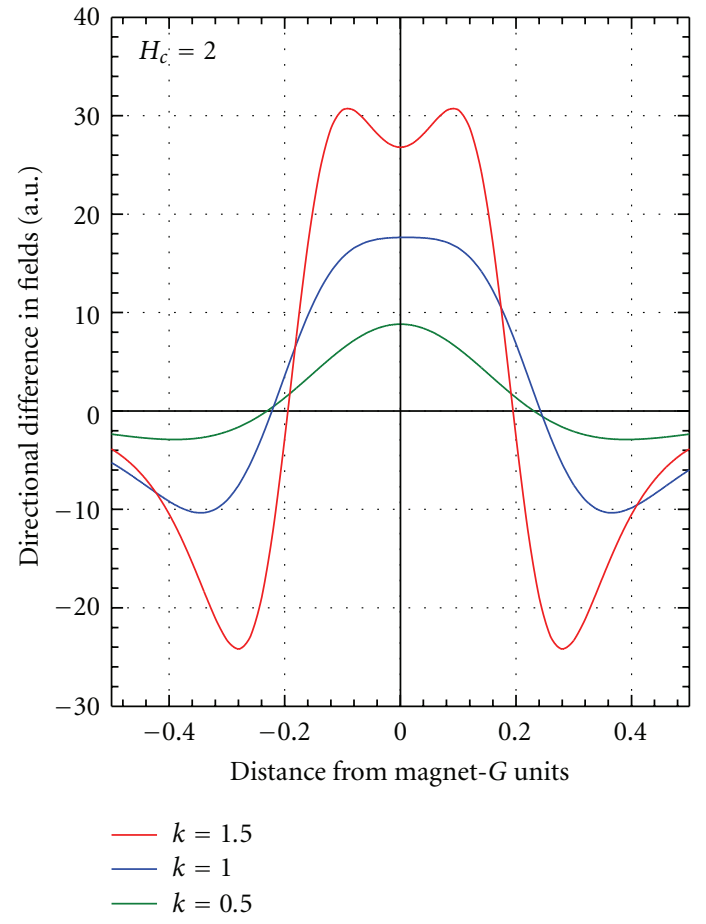

(b)

Figure 6: (a) Hysteresis Loops for constant $H_{c}$, variable $k$ values. (b) Resulting $D(x)$.

Major hysteresis loops created from (4) and (5) for the values of $H_{c}$ and $k$ indicated are shown in Figures $6(\mathrm{a})$ and $7(\mathrm{a})$. Resultant values of $D(x)$ are shown in Figures 6(b) and $7(\mathrm{~b})$. The sizes of the central positive peak and the symmetrical negative peaks are seen to vary directly with the loop squareness, peak $M$, and $H_{c}$, as does hysteresis loss.

\section{Experimental}

An experimental apparatus was set up on a vertical milling machine, thereby conveniently accommodating a variety of experimental conditions, including SUT size (width, thickness, and length), field sensor position relative to the SUT edge, length of Stroke and center position relative to the field sensor, adjustability of both the space between the magnet face and SUT (dimension $b$ in Figure 1), and the location of the magnet relative to the field sensor centerline. An Allegro 3515 UA Hall effect field sensor (sensitivity = $0.0628 \mathrm{mV} / \mathrm{A} / \mathrm{m}$ ), with $d$ (Figure 1$)=1.62 \mathrm{~mm}$, was mounted just below $(\sim 0.05 \mathrm{~mm})$ the mounting surface of the SUT. $\mathrm{Nd}-\mathrm{Fe}-\mathrm{B}$ magnets of a variety of sizes and energy levels were mounted into aluminum holders having common mounting features (a $9.5 \mathrm{~mm}$ diameter $\times 20 \mathrm{~mm}$ long cylindrical portion) to allow easy interchangeability in the machine spindle.

Except that the magnet was stationary while the SUT/ field sensor combination (being mounted on the milling machine table) were the movable elements, operation of the apparatus followed the description in the Introduction.
For each magnet and gap combination, $H_{L}(x)$ was first measured without any SUT. The comparative amplitudes, $\pm H_{L \text { Peak }}$ for the various magnet/"gap" ( $G$ in Figure 1 ) combinations were used to gain understanding on how these parameters affect the later test results. On the basis of the plot of (1) in Figure 3, together with assumption 2, $G$ was presumed to be the distance between $\pm H_{L P e a k}$. Measurements of this distance with two or more different physical gaps allowed both the location of the equivalent dipole ("a" in Figure 1(b)) and the dipole moment to be calculated.

Values of $D(x)$, calculated from measurements of $H_{\mathrm{F}}(x)$ and $H_{\mathrm{R}}(x)$ for the samples and magnet indicated, are plotted in Figures 8 and 9. The most salient features of the plots are seen to match the prediction of the analysis. Moreover, since losses in electrical steels are in approximate proportion to their grade number, and the drastic reduction in coercivity by annealing of cold worked steels is common knowledge, these plots verify the theoretically expected correlation between the amplitude of the signature features and the factors contributing to hysteresis loss. The nominal coercivity values of the three samples shown in Figure 8 are $100 \mathrm{~A} / \mathrm{m}, 45 \mathrm{~A} / \mathrm{m}$, $35 \mathrm{~A} / \mathrm{m}$ for the $800-50,350-50$, and the 290-50, respectively. Comparison with Figure 8 teaches us that there is indeed a larger difference between the 800-50 and the 350-50 than between the 290-50 and the 350-50.

The versatility of the described method was shown by its use to measure the relative losses in regions near the cut edges of strip samples of the 350 and 800 grades. The SUT was placed with the edge being examined $\sim 1 \mathrm{~mm}$ over the center 


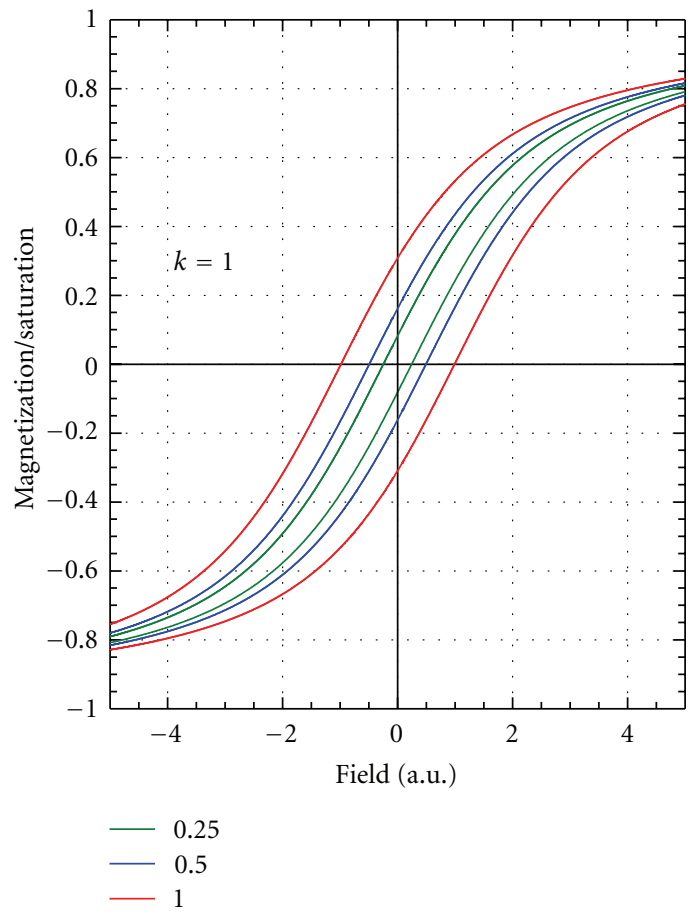

(a)

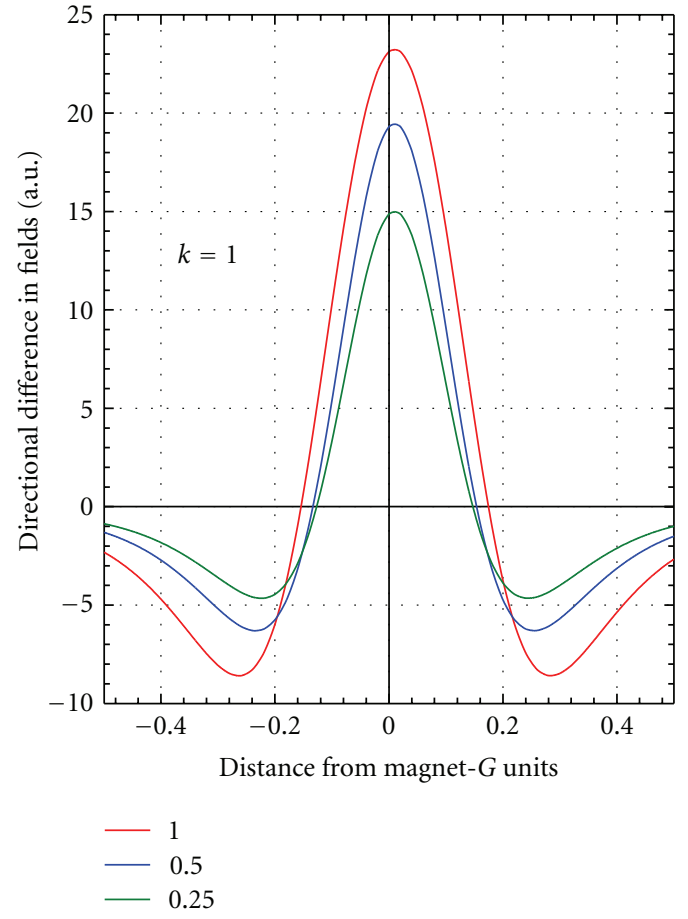

(b)

Figure 7: (a) Hysteresis Loops for constant $k$, variable $H_{c}$ values. (b) Resulting $D(x)$.

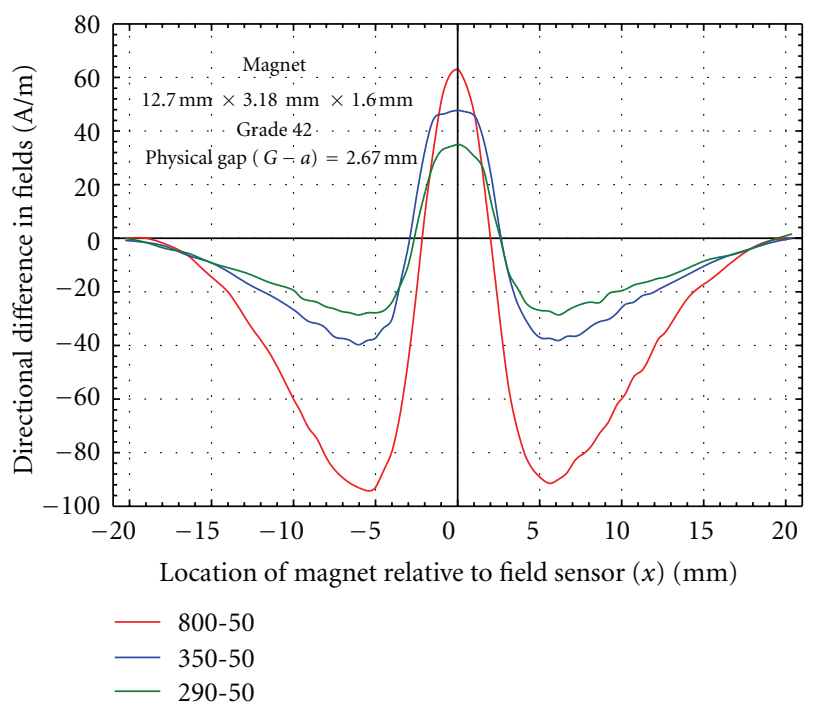

Figure 8: $D(x)$ from measured values of $H_{\mathrm{F}}(x)$ and $H_{\mathrm{R}}(x)$ for the 3 grades of NO Si steels indicated.

of the field sensor. A magnet, $3.18 \mathrm{~mm}$ square by $12.7 \mathrm{~mm}$ long (in the direction of $m$ ) was positioned $1 \mathrm{~mm}$ back from the edge and $2.5 \mathrm{~mm}$ above the SUT. $D(x)$ measurements on 2 samples each, cut by a fast moving laser, averaged $40 \%$ (350 grade) and $14 \%$ ( 800 grade) less than those cut more slowly, in good agreement with results from other measurement methods [4].

The more compact spacing of the signature features predicted in the analysis than those experimentally observed

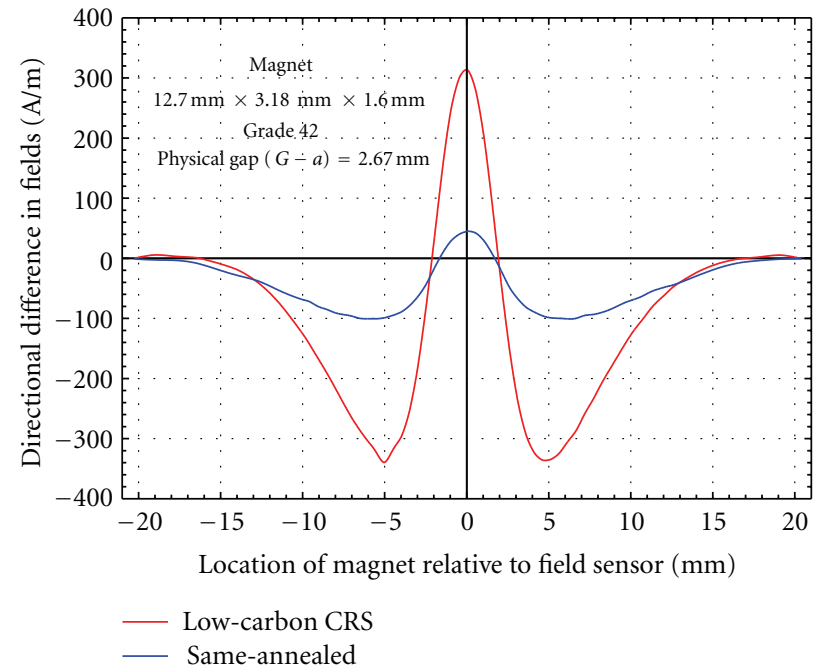

FIGURE 9: Same as Figure 8 for $0.61 \mathrm{~mm}$ thick cold rolled AISI 1010 in as-received and annealed conditions.

is attributed to several critical but enormously complicating factors being ignored in the analysis; namely, the interaction between the magnetization and the generated fields and the gradients in these throughout the SUT thickness. Nevertheless, the model correctly shows that the magnetization gradients are different for the two directions of motion, a difference founded on the double-valued $M-H$ relationships which manifest "hysteresis." We also ignored in this paper the effects of sample thickness, distance of the field sensor from 
the sample surface, possible effects of the circuit permeance on the field from the magnet, and so forth. However, a significant contributor to the less compact detected field difference profile is this interaction of the $\nabla \cdot M$ fields with the $M$ fields.

This paper proposes an experimental method for locally assessing hysteresis properties, and a theoretical basis is given for explaining the obtained difference curves. The correlation between the experimentally obtained Figure 8 and Figures $7(\mathrm{a})$ and 7(b) shows that indeed the difference curves are correlated with the hysteresis losses. The same correlation was observed with Figure 9. The results in this paper are qualitative, and by refinement of the numerical model for explaining the difference curves we can aim in further work to more quantitative assessment of hysteresis properties and losses. The numerical model can be refined by, for example, taking into account the thickness of the sample under study so to have a more correct explanation of the measurements. The method we describe does give quantitative results. We do not claim, however, that the quantitative description of salient features of $D(x)$ plots have translatable correlations with quantitative descriptions of salient features of $\mathrm{B}-\mathrm{H}$ plots.

A possibility to more quantitative studies is to determine the input model parameters (i.e., $k, H_{c}$, and $f$ ) starting from directly measured B-H loops. Also, more advancements are needed in the theory for better understanding the $D(x)$ measurements. For instance, sensitivity analysis can help us in better understanding the measurements by looking at how the variation in one parameter affects the $D(x)$.

\section{Conclusions}

In this paper, we propose a unique methodology together with its modus operandi and important experimental results. Both analytical and experimental results convincingly show the signature features of the difference measurements $D(x)$ to be reflective of those same properties of test sample material, which are the underlying determinants of the size, shape, and intercept features of conventional hysteresis loops. As such, and considering both the rapidity and sensitivity by which $D(x)$ measurements might be obtained with purposefully constructed apparatus of obvious simplicity, the utility of the described method for comparative evaluations by nondestructive means, of factors affecting hysteresis properties, seems well established. The proposed technique has the possibility to assess in detail the relationships between microstructure (or internal stress) and magnetic performance.

\section{References}

[1] M. Emura, M. F. de Campos, F. J. G. Landgraf, and J. C. Teixeira, "Angular dependence of magnetic properties of $2 \%$ silicon electrical steel," Journal of Magnetism and Magnetic Materials, vol. 226-230, part 2, pp. 1524-1526, 2001.

[2] E. G. Araujo, J. Schneider, K. Verbeken, G. Pasquarella, and Y. Houbaert, "Dimensional effects on magnetic properties of fesi steels due to laser and mechanical cutting," IEEE Transactions on Magnetics, vol. 46, no. 2, pp. 213-216, 2010.

[3] G. Crevecoeur, P. Sergeant, L. Dupré, L. Vandenbossche, and R. Van de Walle, "Analysis of the local material degradation near cutting edges of electrical steel sheets," IEEE Transactions on Magnetics, vol. 44, no. 11, pp. 3173-3176, 2008.

[4] I. J. Garshelis, G. Crevecoeur, S. P. L. Tollens, and L. Dupre, "Application of the drag force method to evaluate magnetic property degradation near the cut edges of electrical steels," Journal of Applied Physics, vol. 109, no. 7, Article ID 07E518, 2011.

[5] B. D. Cullity, Introduction to Magnetic Materials, AddisonWesley, Reading, Mass, USA, 1972. 

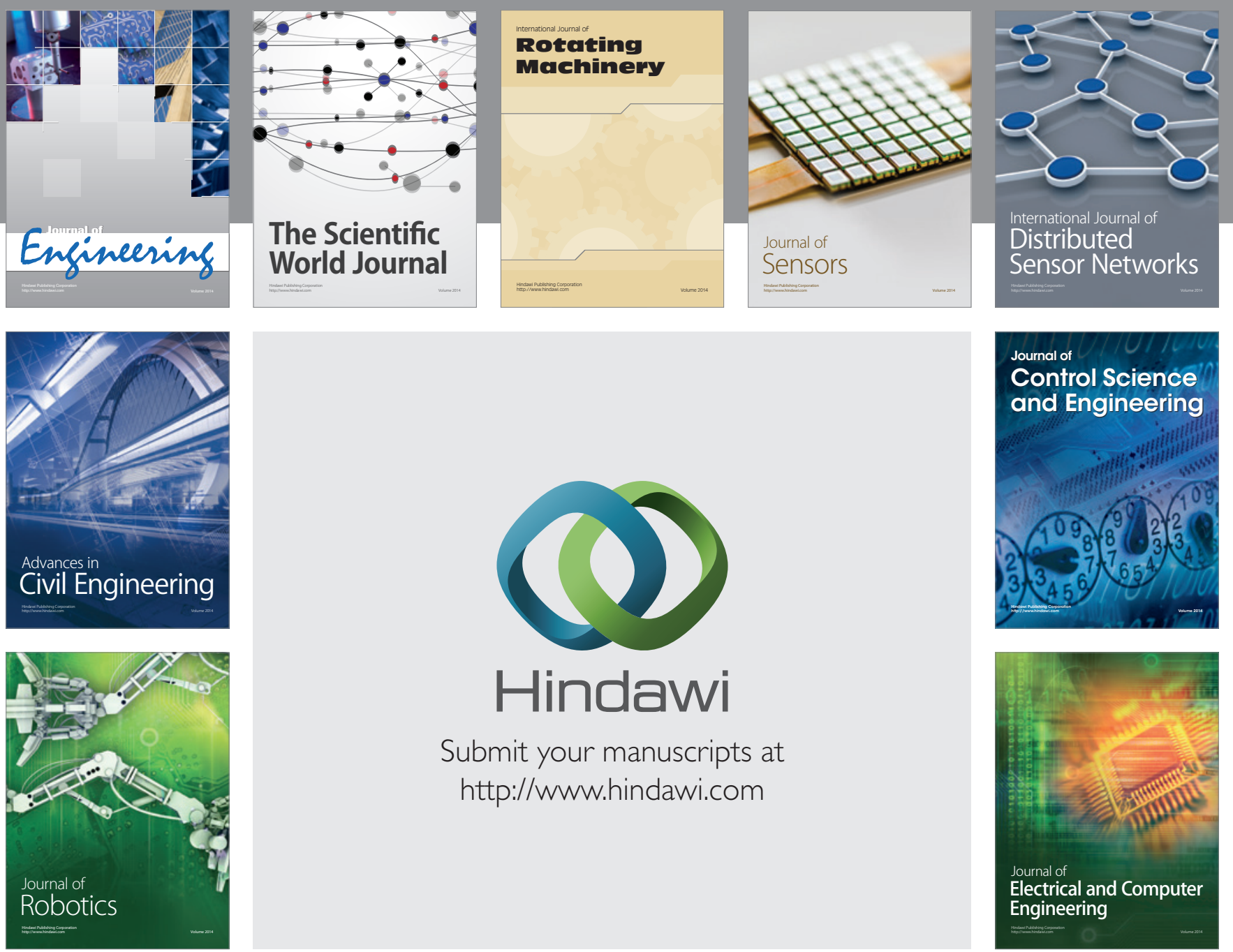

Submit your manuscripts at

http://www.hindawi.com
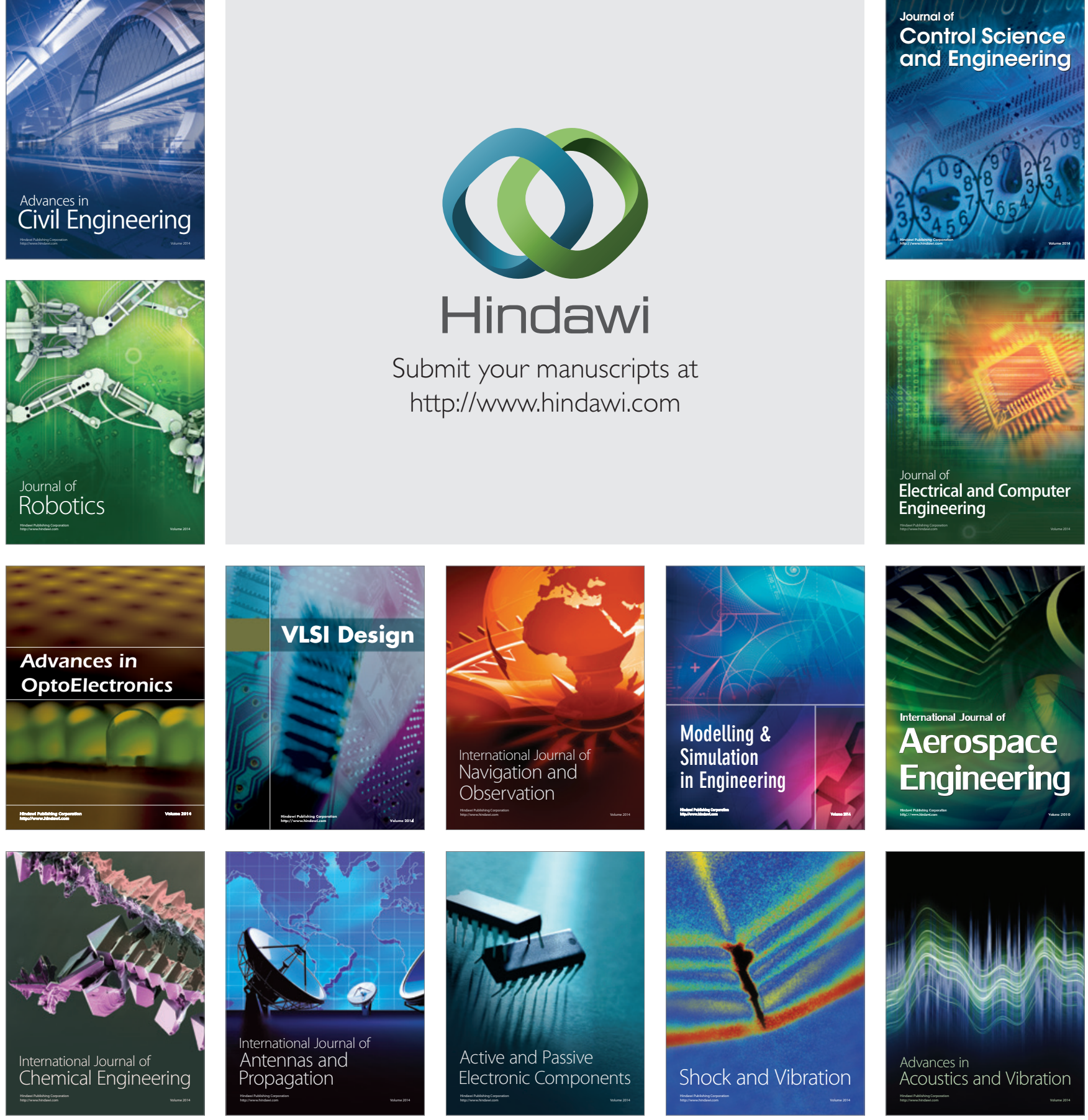